\title{
Relationship between the structure and composition of rumen microorganisms and the digestibility of neutral detergent fibre in goats
}

\author{
Kaizhen Liu', Lizhi Wang ${ }^{1, *}$, Tianhai Yan ${ }^{1,2}$, Zhisheng Wang' ${ }^{1}$, Bai Xue', and Quanhui Peng'
}

\section{* Corresponding Author: Lizhi Wang Tel: +86-835-2885165, Fax: +86-835-2885065 \\ E-mail: wanglizhi08@aliyun.com}

'Animal Nutrition Institute, Key Laboratory of Bovine Low-Carbon Farming and Safe Production, Sichuan Agricultural University, Chengdu, Sichuan 611130 China

${ }^{2}$ Agri - Food and Biosciences Institute, Hillsborough, Co. Down, BT26 6DR, UK

\section{ORCID}

Kaizhen Liu

https://orcid.org/0000-0002-1364-0061 Lizhi Wang

https://orcid.org/0000-0003-3074-4273

Tianhai Yan

https://orcid.org/0000-0002-1994-5202

Zhisheng Wang

https://orcid.org/0000-0001-7999-3612

Bai Xue

https://orcid.org/0000-0001-9283-3015

Quanhui Peng

https://orcid.org/0000-0003-1421-9145

Submitted Jan 11, 2018; Revised May 3, 2018; Accepted Jun 7, 2018
Objective: This experiment was conducted to compare the structure and composition of ruminal microorganisms in goats with high and low neutral detergent fibre (NDF) digestibility. Methods: Nineteen crossbred goats were used as experimental animals and fed the same total mixed rations during the 30-day pre-treatment and 6-day digestion trialperiods. All faeces were collected during the digestion period for measuring the NDF digestibility. Then, high and the low NDF digestibility individuals were chosen for the high NDF digestibility group (HFD) and low NDF digestibility group (LFD), respectively. Rumen contents were collected for total microbial DNA extraction. The V4 region of the bacterial $16 \mathrm{~S}$ rRNA gene was amplified using universal primers of bacteria and sequenced using high-throughput sequencer. The sequences were mainly analysed by QIIME 1.8.0.

Results: A total of 18,694 operational taxonomic units were obtained, within $81.98 \%$ belonged to bacteria, $6.64 \%$ belonged to archaea and $11.38 \%$ was unassigned microorganisms. Bacteroidetes, Firmicutes, and Proteobacteria were the predominant microbial phyla in both groups. At the genus level, the relative abundance of fifteen microorganisms were significantly higher $(\mathrm{p}<0.05)$ and six microorganisms were extremely significantly higher $(\mathrm{p}<0.01)$ in LFD than HFD. Overall, 176 core shared genera were identified in the two groups. The relative abundance of 2 phyla, 5 classes, 10 orders, 13 families and 15 genera had a negative correlation with NDF digestibility, but only the relative abundance of Pyramidobacter had a positive correlation with NDF digestibility.

Conclusion: There were substantial differences in NDF digestibility among the individual goats, and the NDF digestibility had significant correlation with the relative abundance of some ruminal microorganisms.

Keywords: Goats; Neutral Detergent Fibre Digestibility; High-throughput Sequencing; Rumen Microorganism

\section{INTRODUCTION}

Fibre accounts for a high proportion of the diets of ruminants, and it is also an essential nutrient for ruminants. Therefore, the efficiency of feed utilization is significantly influenced by the dietary fibre digestibility in ruminants. It is well known that ruminants themselves can not secrete cellulolytic enzyme. Rather, it is ruminal microorganisms that play a crucial role in the degradation of fibre [1]. To enhance fibre digestibility, the vital step is to obtain a deep understanding of the microorganisms in the rumen.

In a previous report, Fibrobacter succinogenes, Ruminococcus flavefaciens, and Ruminococcus albus were considered to be the most important cellulolytic bacteria in the rumen [2]. However, many recent studies showed that Ruminococcus and Fibrobacter succinogenes had a very low relative abundance in the rumen $[3,4]$, so we doubted that the role of these bacteria in 
the fibre degradation would be amplified. Further, many other important microorganisms associated with fibre digestion might have been ignored. Because previous studies of ruminal microorganisms were entirely dependent on culture techniques and small-scale sequencing, less than $10 \%$ of all microorganisms in the samples could be discovered.

Recently, next-generation sequencing technology had been extensively applied in the study of microbial ecology [4-6]. Compared to culture and fingerprint technologies, high-throughput sequencing can process millions of sequenced reads and obtain the biological information of many microorganisms simultaneously in samples. Inthis study, high-throughput sequencing was firstly applied to investigate the structure and composition of microorganisms of the rumen in goats that had the same genetics, age and management conditions but different neutral detergent fibre (NDF) digestibility.

\section{MATERIALS AND METHODS}

\section{Animal care}

The animal experimental procedures were approved by the Animal Policy and Welfare Committee of the Agricultural Research Organization of Sichuan Province, China, and were in accordance with the guidelines of the Animal Care and Ethical Committee of the Sichuan Agricultural University.

\section{Experimental animals and sampling}

Nineteen castrated Boer crossbred goats (Jianchang black goat and Boer goat crossbreeds) with an average age of 1.5 years were used in this study. The average live body weight of the animals was $41.60 \pm 2.63 \mathrm{~kg}$ at the beginning of the study. All goats were fed the same total mixed rations containing $70 \%$ forage and 30\% concentration (Table 1), and animals were fed at a restricted level of 3.5\% body weight at 08:00, 13:00, and 18:00 hours. Each goat was housed in separate pens with free access to water. The trial lasted for 36 days, including 30 days of adaptation and 6 days of digestion period. Every day during the digestion period, all faeces were collected, and $10 \%$ of them were sampled randomly and then mixed with a $10 \%$ volume of $10 \% \mathrm{HCl}$ for nitrogen fixation. At the same time, the daily feed intake and residual intake were recorded over six days for the subsequent calculation of nutritional composition and digestibility.

After digestion trials, the next morning before feeding, 50 $\mathrm{mL}$ of rumen contents from each goat were sampled using oral stomach tubes attached to an electric pump as previously described [7]. The rumen contents were repeatedly flapped on ice to ensure that the microbes associated with the feed particles were fully immersed in liquid. Then, the rumen contents were strained using four layers of gauze, and the rumen fluid was collected and aliquoted into $10-\mathrm{mL}$ centrifuge tubes. Rumen liquid samples were sealed and stored at $-80^{\circ} \mathrm{C}$ until
Table 1. The composition and nutritional ingredients of the diet

\begin{tabular}{lc}
\hline Items & Content \\
\hline Ingredients (\%, DM basis) & 35 \\
Alfalfa meal & 35 \\
Rice straw & 2.67 \\
Corn & 8.33 \\
Rice & 5.0 \\
Soybean meal & 13 \\
Wheat bran & 0.5 \\
Premix ${ }^{1)}$ & 0.5 \\
NaCl & 100 \\
Total & \\
Nutrition levels & \\
Metabolizable energy (MJ/kg) & 7.7 \\
Crude protein & 10.21 \\
Neutral detergent fibre & 46.13 \\
Calcium & 0.92 \\
Phosphorus & 0.31 \\
\hline 1) Premix provides the following nutrients per kg of the diet: Fe (as ferrous sulfate) \\
30 mg; Cu (as copper sulfate) 10 mg; Zn (as zinc sulfate) 50 mg; Mn (as manga- \\
nese sulfate) 60 mg; Vit A 2,937 IU; Vit D 343 IU dry matter; Vit E 30 IU. \\
2) Nutrition levels are values of measurement except that ME is a value from a \\
calculation.
\end{tabular}

DNA extraction.

\section{Analysis of samples and grouping}

All samples of feed and faeces were dried in a forced-air oven at $65^{\circ} \mathrm{C}$ for $48 \mathrm{~h}$ to measure the dry matter $(\mathrm{DM})$ and then ground to pass through a 40-mesh sieve. NDF and acid detergent fibre (ADF) in samples were determined using the filter bag technology without sodium sulphite, and expressed with residual ash. Ether extract (EE) was determined by Soxhlet extraction method, crude protein $(\mathrm{CP})$ was determined by the Kjeldahl method, organic matter (OM) was measured in a muffle furnace at $550^{\circ} \mathrm{C}$ for 6 hours, and calcium and phosphorus were measured using an atomic absorption spectrophotometer [8].

The NDF digestibility was calculated as follows [5]:

$$
\operatorname{NDF} \text { digestibility }(\%)=(A \times B-C \times D) / A \times B \times 100
$$

Where A was the feed intake computed as the amount of given feed minus the residual intake during a 6-day digestion period; $B$ was the NDF concentration in the feed; $C$ was the total amount of faeces based on DM in the 6-day digestion period; and D was the NDF concentration in faeces. The mean and standard deviation (SD) of NDF digestibility of all goats were calculated using Excel software. Then, standard deviations above and below the mean were used to group animals into high NDF digestibility (HFD, NDF digestibility $>$ mean+ $0.5 \times \mathrm{SD}$ ) group and low NDF digestibility (LFD, NDF digestibility $<$ mean $-0.5 \times \mathrm{SD}$ ) group as the previous described method [9]. 


\section{DNA extraction}

Total DNA was extracted from each rumen sample of the HFD and LFD groups using the TIANamp Bacteria DNA Kit (TIANGEN, Peking, China) according to the manufacturer's guidelines as described before. Then, the quality and quantity of the DNA samples was determined through agarose electrophoresis and a Nanodrop 8000 Spectrophotometer (Thermo Fisher Scientific, Brisbane, Australia) respectively.

\section{Polymerase chain reaction amplification and sequencing}

The universal primers of bacteria (341F:5'-CCTAYGGGRBGCA SCAG-3' and A806R:5'--GGACTACHVGGGTWTCTAAT-3') were used to amplify the V4 hyper variable regions of $16 \mathrm{~S}$ rRNA [10]. All polymerase chain reaction (PCR) amplifications were performed in triplicate with $50 \mu \mathrm{L}$ of reactions volume (PCR thermal cycler Model C1000, Bio-rad, Richmond, CA, USA) that consisted of $1.25 \mu \mathrm{L}$ of each primer (10 $\mu \mathrm{mol} / \mathrm{L}), 1 \mu \mathrm{L}$ of $10 \mathrm{mmol} / \mathrm{L}$ dNTP Mixture, $5 \mu \mathrm{L}$ of $10 \times$ ExTaq buffer (20 mmol/ $\mathrm{L} \mathrm{Mg}^{2+}$; TaKaRa Inc, Dalian, China), $1 \mu \mathrm{L}$ of $50 \mathrm{ng} / \mu \mathrm{L}$ template DNA, $0.25 \mu \mathrm{L}$ of $5 \mathrm{U} / \mu \mathrm{L}$ Taq DNA polymerase ( $\mathrm{Mg}^{2+}$ plus, Takara Inc., China) and distilled water to a final volume of $50 \mu \mathrm{L}$. The amplification was initiated with a denaturation at $94^{\circ} \mathrm{C}$ for $3 \mathrm{~min}, 30$ cycles of denaturation at $94^{\circ} \mathrm{C}$ for $30 \mathrm{~s}, 58^{\circ} \mathrm{C}$ for $30 \mathrm{~s}, 72^{\circ} \mathrm{C}$ for $90 \mathrm{~s}$, and a final extension at $72^{\circ} \mathrm{C}$ for $5 \mathrm{~min}$. Finally, the three replicates of DNA extracted from each sample were mixed together.

The products were purified using a PCR Clean-Up system (Promega, Madison, WI, USA) with a purification kit (QIAGEN, Adelaide, Australia) and quantified using a QuantiFlour-ST fluorometer (Promega, USA) [8]. Subsequently, the amplicons of each reaction mixture were pooled into a single tube in equimolar ratios to generate the amplicon libraries. Before the samples were pooled with equal volumes, each amplicon library was firstly diluted to $1 \times 10^{9}$ molecules $/ \mathrm{mL}$. Then the pooled amplicon libraries were diluted to $1 \times 10^{7}$ molecules/mL. Finally, the samples were sent to Macrogen Inc. (Seoul, Korea) and sequenced on an Illumina MiSeq 300PE Sequencing Platform (Novogene, Beijing, China).

\section{Sequencing analysis}

The QIIME pipeline software (version 1.8.0) was used to analyse the reads acquired from Macrogen Inc [11]. Low-quality sequences, such as sequences containing uncertain nucleotides, continuous three nucleotides with $Q$ values less than 20 and unmatched barcode sequences, were removed. Chimeric sequences were removed using Usearch V7.0 based on the Uchime algorithm implemented in QIIME [12]. The clean and high-quality sequences were then clustered into operational taxonomic units (OTUs) based on 97\% similarity. The most abundant sequence was selected as the representative for each OTU and then aligned against the Greengenes data- base (http://greengenes.lbl.gov) using PyNAST [13]. Taxonomic OTU assignments were performed using the RDP Classifier (http://rdp.cme.msu.edu) [4].

Alpha diversity indices (Shannon-Wiener, PD_whole_ tree, Chaol and The observed-species) were computed at the depth of 21,854 sequences. Beta diversity was visualized using principal co-ordinate analysis (PCoA), as measured using an unweighted UniFrac distance matrix. In addition, genera that were shared by all the samples were selected to create a heat map using the $\mathrm{R}$ version 3.0.2 software program [14]. All sequence data in the present study were deposited in the sequence read archive (SRA) of the NCBI database under the number PRJNA290544.

\section{Statistical analysis}

An unpaired two-tailed t-test was performed usingSPSS Statistics software v. 19.0 (IBM, Armonk, NY, USA) to assess the differences of NDF digestibility and microbial relative abundance between the HFD and LFD groups. The results were shown as means \pm SD. The correlation analysis between the relative abundance of bacteria and NDF digestibility was performed. The significant and extremely significant levels were set at $\mathrm{p}<0.05$ and $\mathrm{p}<0.01$, respectively.

\section{RESULTS}

\section{NDF digestibility}

The average NDF digestibility of nineteen goats (Table 2) was $58.83 \% \pm 5.36 \%$ (mean \pm SD). Then, HFD (NDF digestibility $>$ mean $+0.5 \times \mathrm{SD}, \mathrm{n}=5$ ) and LFD (NDF digestibility $<$ mean $-0.5 \times$ $\mathrm{SD}, \mathrm{n}=5$ ) individuals were chosen. The difference in NDF digestibility between the groups was extremely significant $(\mathrm{p}<$ 0.001). There were no significant differences in the apparent digestibility of EE, CP, and ADF between the groups, but there were significant differences in the apparent digestibility of DM, OM between the groups. Comparisons of apparent digestibility of dietary nutrients of the two groups are shown in Supplementary Table S1.

\section{Analysis of the Illumina MiSeq sequencing data of the two groups}

Sequencing depth and alpha diversity: A total of 569,887 sequences were generated from the samples of all goats after quality control with a mean of 56,988 $\pm 24,441$ (mean \pm SD, $n=$ 10). A total of 18,694 OTUs were identified at the $97 \%$ similarity level with a mean of 5,027 $\pm 1,059($ mean $\pm S D, n=10)$. Distribution of valid sequences and OTUs were presented in Supplementary Figure S1 and S2. Alpha diversity was presented in Table 3. The Shannon-Wiener index, Chao I index, the Observed-species and PD_whole_tree had no significant difference between the LFD and HFD groups. To evaluate the depth of sampling, rarefaction curves produced from se- 
Table 2. The NDF digestibility of the diets of 19 Boer goats

\begin{tabular}{lc}
\hline Goats number & NDF digestibility $(\%)$ \\
\hline 1 & 51.24 \\
2 & 51.48 \\
3 & 51.67 \\
4 & 52.11 \\
5 & 55.23 \\
6 & 56.96 \\
7 & 57.03 \\
8 & 57.43 \\
9 & 57.69 \\
10 & 57.72 \\
11 & 58.14 \\
12 & 59.35 \\
13 & 61.52 \\
14 & 61.03 \\
15 & 62.55 \\
16 & 64.60 \\
17 & 65.52 \\
18 & 67.33 \\
19 & 69.24 \\
Mean & 58.83 \\
SD & 5.36 \\
\hline
\end{tabular}

NDF, neutral detergent fibre; SD, standard deviation.

quences and OTUs of bacteria sequencing were shown in Figure 1. The rarefaction curve showed that most samples were nearly asymptotic, which indicated that the depth of sequencing in the present study could cover most of the microorganisms in samples.

\section{Composition of the rumen bacteria}

After the taxonomic summary, all OTUs were classified as 43 phyla, with most of them (81.98\%) belonging to bacteria and
Table 3. The alpha diversity index (calculated at a depth of 21,854 sequences) of microbial communities from LFD and HFD groups (mean $\pm S D, n=5$ )

\begin{tabular}{lccc}
\hline Items & HFD & LFD & p value \\
\hline PD_whole_tree & $223.5 \pm 5.9$ & $223.1 \pm 15.5$ & 0.966 \\
Chao 1 & $5,761.2 \pm 692.3$ & $6,008.7 \pm 297.5$ & 0.484 \\
Shannon & $8.7 \pm 0.4$ & $8.7 \pm 0.4$ & 0.790 \\
The observed-species & $3,267 \pm 692$ & $6,009 \pm 297$ & 0.484 \\
\hline
\end{tabular}

LFD, low neutral detergent fibre digestibility group; HFD, high neutral detergent fibre digestibility group; SD, standard deviation.

only $6.64 \%$ were archaea. The 17 phyla whose relative abundance was more than $0.1 \%$ in all samples were shown in Figure 2 , and the other 26 phyla were not shown because of their low relative abundance. The relative abundance of Nitrospirae ( $\mathrm{p}$ $=0.030)$ and Crenarchaeota $(\mathrm{p}=0.011)$ in HFD group was significantly lower than that in LFD group. The three most predominant phyla were Bacteroidetes $(48.57 \% \pm 4.84 \%$ in HFD; $41.11 \% \pm 10.07 \%$ in LFD), Firmicutes $(15.93 \% \pm 2.94 \%$ in HFD; $17.33 \% \pm 5.85 \%$ in LFD) and Proteobacteria (11.37\% \pm $8.21 \%$ in HFD; $11.08 \% \pm 2.46 \%$ in LFD) in the two groups.

At the class level, Bacteroidia was the most predominant bacterium for all samples, and its average relative abundance reached $44.19 \%$. The relative abundance of Gammaproteobacteria, Bacilli, Acidobacteria- 6 and Nitrospira were significantly different, Thaumarchaeota and PBS-25 were extremely significantly different between the two groups. The most predominant order-level bacterium was Bacteroidales, which was one of the most important orders in phylum Bacteroidia. And the predominant bacterial family was Prevotellaceae (21.06\%).

At the genus level, Prevotella was the most predominant taxa both in the two groups (20.98\%). However, the relative abundance of Prevotella had no significant difference between the two groups. The comparisons of taxa relative abundance

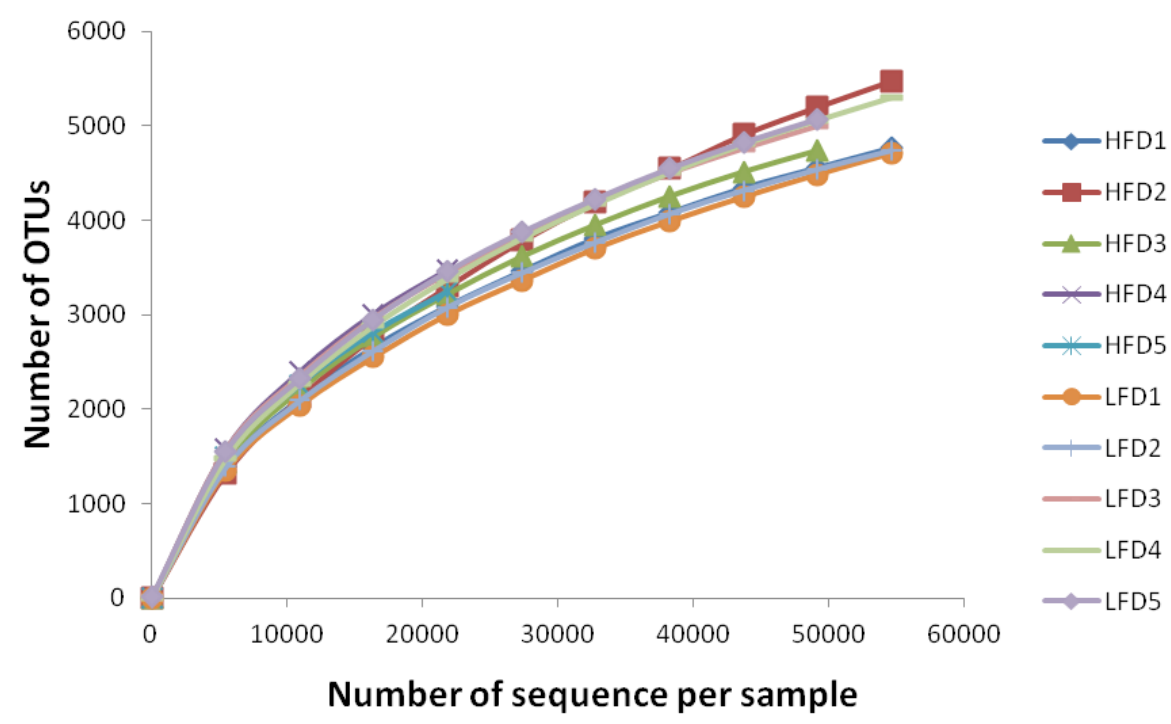

Figure 1. Rarefaction curves of each sample in the high neutral detergent fibre digestibility group and low neutral detergent fibre digestibility group. 


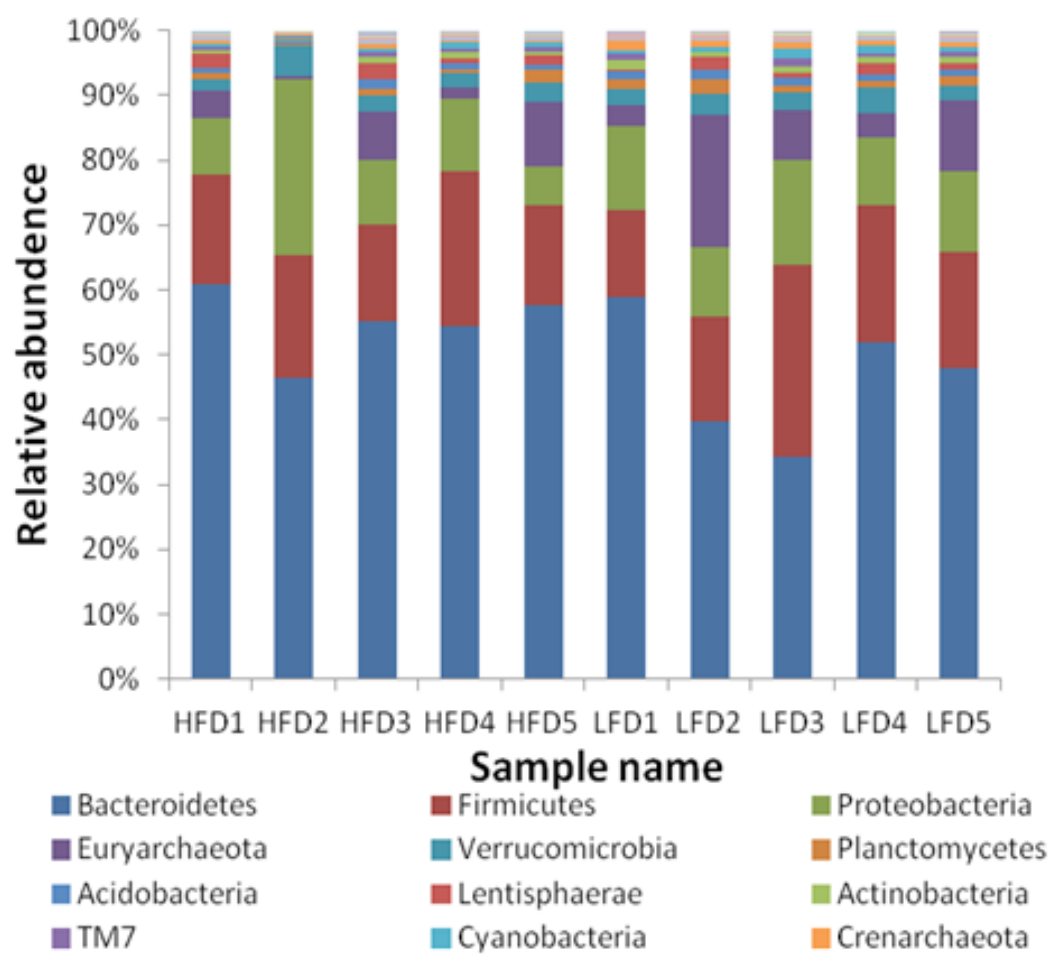

Figure 2. Taxonomic composition of the rumen bacterial communities on the phylum level.

from the levels of phylum to genus were presented in Table 4.

\section{Shared genera}

A total of 176 genera were shared by all goats, and these genera accounted for $85.20 \%$ of all taxa. There were 197 genera shared by the goats of HFD group and 227 genera shared by the goats of LFD group. These shared genera and phylogenetic placements among individuals were presented in a heatmap (Figure 3).

\section{Clustering dissimilarity analysis}

The unweighted UniFrac distance matrix among samples was measured, and the PCoA plot based on unweighted UniFrac distance metric was generated according to the OTU table (Figure 4). The closer the distance between points was, the higher similarity in the community structure was between two samples. The results showed that the bacterial community composition between groups and samples had no clear dissimilarity because the spots of the two groups were not clearly separated. Moreover, PC1 and PC2 components only contributed to $14.39 \%$ and $12.51 \%$ of the variability, respectively.

\section{Relationship between rumen microorganisms and NDF digestibility}

The relative abundance of Crenarchaeota at the phylum level, Thaumarchaeota and PBS-25 at the class level, Nitrososphaerales, SBR1031 and PBS-25 (Class) at the order level,
Nitrososphaeraceae, Sphingomonadaceae, ${ }^{*}$ Lactobacillales (Other), Nitrospiraceae and SJA-101 at the family level, Candidatus Nitrososphaera, ${ }^{\star}$ Flavobacteriaceae (Family), ${ }^{\star}$ SJA101 (Family), ${ }^{\star}$ Rhizobiaceae (Family), ${ }^{\star}$ Hyphomonadaceae (Family), and Nitrosopumilus at the genus level had extremely significantly negative relationship with NDF digestibility ( $\mathrm{p}<$ 0.01 ). Furthermore, there were also one phylum-level, three class-level, seven order-level, eight family- level and nine genuslevel bacteria had significant negative relationships with NDF digestibility $(\mathrm{p}<0.05)$. Only Pyramidobacter had positive correlation with NDF digestibility $(\mathrm{p}<0.05)$ (Table 5$)$.

\section{DISCUSSION}

The previous studies reported that the NDF digestibility in ruminants was mainly affected by physical and chemical pretreatment methods of roughage, diet composition and additives [15-17]. It was generally believed that there was no significant difference in the digestibility of NDF in animals under the same conditions. However, in the present study, although experimental goats had the same genetic background, gender, and age were fed the same feed, obvious differences in NDF digestibility among individuals were found, with a wide range of $51.67 \%$ to $69.24 \%$. To date, there have been few reports about the individual differences in NDF digestibility. Nevertheless, significant individual differences in residual feed intake (RFI) had been reported in a number of previous re- 
Table 4. The microorganisms with significant differences between HFD and LFD groups from phylum to genus

\begin{tabular}{|c|c|c|c|c|}
\hline \multirow{2}{*}{ Taxa } & & \multicolumn{3}{|c|}{ Relative abundance (\%) } \\
\hline & & $\operatorname{HFD}(n=5)$ & $\operatorname{LFD}(n=5)$ & $p$ value \\
\hline \multirow[t]{2}{*}{ Phylum } & Crenarchaeota & $0.335 \pm 0.178$ & $0.850 \pm 0.299$ & 0.011 \\
\hline & Nitrospirae & $0.047 \pm 0.013$ & $0.089 \pm 0.033$ & 0.030 \\
\hline \multirow[t]{6}{*}{ Class } & Gammaproteobacteria & $4.934 \pm 1.022$ & $7.693 \pm 2.196$ & 0.034 \\
\hline & Bacilli & $3.542 \pm 1.080$ & $5.945 \pm 1.767$ & 0.032 \\
\hline & Thaumarchaeota & $0.323 \pm 0.179$ & $0.836 \pm 0.274$ & 0.008 \\
\hline & Acidobacteria-6 & $0.349 \pm 0.157$ & $0.562 \pm 0.063$ & 0.023 \\
\hline & Nitrospira & $0.047 \pm 0.013$ & $0.089 \pm 0.033$ & 0.030 \\
\hline & PBS-25 & 0.000 & $0.002 \pm 0.001$ & 0.000 \\
\hline \multirow[t]{12}{*}{ Order } & Enterobacteriales & $3.559 \pm 0.780$ & $5.756 \pm 1.626$ & 0.026 \\
\hline & Lactobacillales & $2.514 \pm 0.681$ & $4.341 \pm 1.038$ & 0.011 \\
\hline & Nitrososphaerales & $0.311 \pm 0.172$ & $0.785 \pm 0.238$ & 0.007 \\
\hline & iii1-15 & $0.331 \pm 0.145$ & $0.538 \pm 0.080$ & 0.023 \\
\hline & Pseudomonadales & $0.332 \pm 0.082$ & $0.459 \pm 0.092$ & 0.050 \\
\hline & Thiotrichales & $0.134 \pm 0.030$ & $0.218 \pm 0.032$ & 0.003 \\
\hline & SBR1031 & $0.052 \pm 0.032$ & $0.161 \pm 0.084$ & 0.027 \\
\hline & [Marinicellales] & $0.043 \pm 0.021$ & $0.096 \pm 0.042$ & 0.035 \\
\hline & MND1 & $0.032 \pm 0.024$ & $0.105 \pm 0.028$ & 0.002 \\
\hline & Nitrospirales & $0.047 \pm 0.013$ & $0.089 \pm 0.033$ & 0.030 \\
\hline & [Entotheonellales] & $0.004 \pm 0.006$ & $0.026 \pm 0.018$ & 0.034 \\
\hline & *PBS-25 (Class) & 0.000 & $0.002 \pm 0.001$ & 0.001 \\
\hline \multirow[t]{19}{*}{ Family } & Enterobacteriaceae & $3.559 \pm 0.780$ & $5.756 \pm 1.626$ & 0.026 \\
\hline & Bacteroidaceae & $2.081 \pm 0.344$ & $3.058 \pm 0.501$ & 0.007 \\
\hline & Enterococcaceae & $1.477 \pm 0.303$ & $2.129 \pm 0.521$ & 0.042 \\
\hline & Lactobacillaceae & $0.800 \pm 0.311$ & $1.869 \pm 0.772$ & 0.021 \\
\hline & Nitrososphaeraceae & $0.311 \pm 0.172$ & $0.785 \pm 0.238$ & 0.007 \\
\hline & *iii1-15 (Order) & $0.270 \pm 0.118$ & $0.419 \pm 0.046$ & 0.029 \\
\hline & Sphingomonadaceae & $0.225 \pm 0.091$ & $0.354 \pm 0.013$ & 0.014 \\
\hline & Piscirickettsiaceae & $0.134 \pm 0.030$ & $0.218 \pm 0.032$ & 0.003 \\
\hline & Pseudomonadaceae & $0.063 \pm 0.025$ & $0.117 \pm 0.032$ & 0.019 \\
\hline & [Marinicellaceae] & $0.043 \pm 0.021$ & $0.096 \pm 0.042$ & 0.035 \\
\hline & *MND1 (Order) & $0.032 \pm 0.024$ & $0.105 \pm 0.028$ & 0.002 \\
\hline & *Rhizobiales (Order) & $0.032 \pm 0.017$ & $0.098 \pm 0.054$ & 0.031 \\
\hline & Ellin517 & $0.034 \pm 0.027$ & $0.071 \pm 0.011$ & 0.023 \\
\hline & *Lactobacillales (Other) & $0.029 \pm 0.014$ & $0.059 \pm 0.014$ & 0.010 \\
\hline & Nitrospiraceae & $0.016 \pm 0.009$ & $0.051 \pm 0.026$ & 0.022 \\
\hline & *Lactobacillales (Order) & $0.014 \pm 0.007$ & $0.032 \pm 0.016$ & 0.050 \\
\hline & SJA-101 & $0.008 \pm 0.009$ & $0.037 \pm 0.016$ & 0.008 \\
\hline & Hyphomonadaceae & $0.009 \pm 0.008$ & $0.022 \pm 0.006$ & 0.022 \\
\hline & Saccharospirillaceae & 0.000 & $0.001 \pm 0.001$ & 0.044 \\
\hline \multirow[t]{22}{*}{ Genus } & *Enterobacteriaceae (Family) & $3.524 \pm 0.770$ & $5.710 \pm 1.623$ & 0.026 \\
\hline & *Enterococcaceae (Other) & $1.182 \pm 0.221$ & $1.750 \pm 0.408$ & 0.026 \\
\hline & Lactobacillus & $0.800 \pm 0.311$ & $1.869 \pm 0.772$ & 0.021 \\
\hline & Bacteroides & $0.915 \pm 0.168$ & $1.299 \pm 0.315$ & 0.040 \\
\hline & Veillonella & $0.501 \pm 0.159$ & $0.804 \pm 0.219$ & 0.037 \\
\hline & CandidatusNitrososphaera & $0.308 \pm 0.171$ & $0.776 \pm 0.244$ & 0.008 \\
\hline & $5-7 N 15$ & $0.334 \pm 0.095$ & $0.561 \pm 0.169$ & 0.031 \\
\hline & Kaistobacter & $0.172 \pm 0.070$ & $0.269 \pm 0.042$ & 0.028 \\
\hline & *Piscirickettsiaceae (Family) & $0.133 \pm 0.030$ & $0.217 \pm 0.032$ & 0.003 \\
\hline & Pseudomonas & $0.054 \pm 0.013$ & $0.098 \pm 0.035$ & 0.032 \\
\hline & * [Marinicellaceae] (Family) & $0.043 \pm 0.021$ & $0.096 \pm 0.042$ & 0.035 \\
\hline & ${ }^{*}$ Flavobacteriaceae (Family) & $0.030 \pm 0.015$ & $0.078 \pm 0.037$ & 0.029 \\
\hline & *Ellin517 (Family) & $0.034 \pm 0.027$ & $0.071 \pm 0.011$ & 0.023 \\
\hline & *SJA-101 (Family) & $0.008 \pm 0.009$ & $0.037 \pm 0.016$ & 0.008 \\
\hline & Pyramidobacter & $0.028 \pm 0.016$ & $0.008 \pm 0.006$ & 0.034 \\
\hline & *Rhizobiaceae (Family) & $0.004 \pm 0.005$ & $0.028 \pm 0.007$ & 0.000 \\
\hline & *Hyphomonadaceae (Family) & $0.007 \pm 0.007$ & $0.022 \pm 0.006$ & 0.007 \\
\hline & Nitrosopumilus & $0.002 \pm 0.004$ & $0.025 \pm 0.012$ & 0.004 \\
\hline & Paraprevotella & $0.002 \pm 0.002$ & $0.007 \pm 0.004$ & 0.046 \\
\hline & *Cenarchaeaceae (Family) & $0.001 \pm 0.002$ & $0.006 \pm 0.004$ & 0.049 \\
\hline & Dyadobacter & 0.000 & $0.001 \pm 0.001$ & 0.048 \\
\hline & Saccharospirillum & 0.000 & $0.001 \pm 0.001$ & 0.044 \\
\hline
\end{tabular}

HFD, high neutral detergent fibre digestibility group; LFD, low neutral detergent fibre digestibility group.

Taxa that could not be assigned to a certain classification but still had significant differences between groups were displayed using the highest taxonomic level at which they could be assigned. In addition, the levels are shown in parentheses and with the superscript "*". The same below. searches [18-20]. RFI was widely used to evaluate the feed utilization efficiency $[21,22]$. Feed utilization efficiency of ruminants depended jointly on the digestibility of various nutrients in feed, including NDF. Furthermore, the concentration of NDF in the diets of goats was usually more than $35 \%$. Therefore, the individual differences in NDF digestibility in the present study were reasonable.

After the comparison analysis, it was found that difference in the NDF digestibility between the LFD $(52.35 \pm 1.64)$ and HFD groups $(65.85 \pm 2.56)(\mathrm{p}<0.000)$ was significantly different in the present work. For ruminants, the rumen is the main site for the digestion of nutrients, especially for fibre. The digestion of fibre depends entirely on symbiotic ruminal microorganisms because the ruminants themselves cannot secrete cellulolytic enzymes [1,23]. Accordingly, the fundamental reason leading to the differences in NDF digestibility between the two groups in this study might due to the differences in the structure and composition of ruminal microorganisms. To prove this hypothesis, Illumina high-throughput sequencing was conducted to compare the microbiome communities between the two groups.

The high-throughput sequencing results indicated that the three predominant phyla in the rumens of all goats were Bacteroidetes, Firmicutes and Proteobacteria, which was consistent with many past studies in herbivores $[6,14]$. At the genus level, the relative abundance of Prevotella was the highest in all samples, and this result was similar to previous studies [24]. Prevotella was also shared by all of the samples and its relative abundance was not significantly difference between the two groups. This above results explained that the member of microbial flora between the two groups was not clearly different, which was also showed in the PCoA plot (Figure 4). It was probably due to the same genetic, age and diets of the experimental animals and the same rear conditions during the trials, which were regarded as the key factors that affected the member of microbial flora by the previous researchers [4, $6,14]$. The difference of microbial flora was mainly presented in the relative abundance of bacteria.

Previous studies indicated that Ruminococcus, Fibrobacter, and Clostridium could produce a large amount of cellulase and hemicellulase in vitro [2]. Therefore, it was believed that these bacteria had an important role in fibre degradation and the higher abundance of them meant the higher fibre digestibility in the rumen. Thisopinion was partially consistent with the results of the present study, in which the relative abundance of Ruminococcus ( $0.60 \%$ in HFD, $0.37 \%$ in LFD), Fibrobacter ( $0.15 \%$ in HFD, $0.11 \%$ in LFD) and Clostridium ( $0.15 \%$ in HFD, $0.10 \%$ in LFD) were all higher in the HFD group than in the LFD group. However, the difference of their relative abundance between groups was not significant, and it was particularly worth noting that the relative abundance of these bacteria was very low. To improve the digestibility of fibre, previous studies 


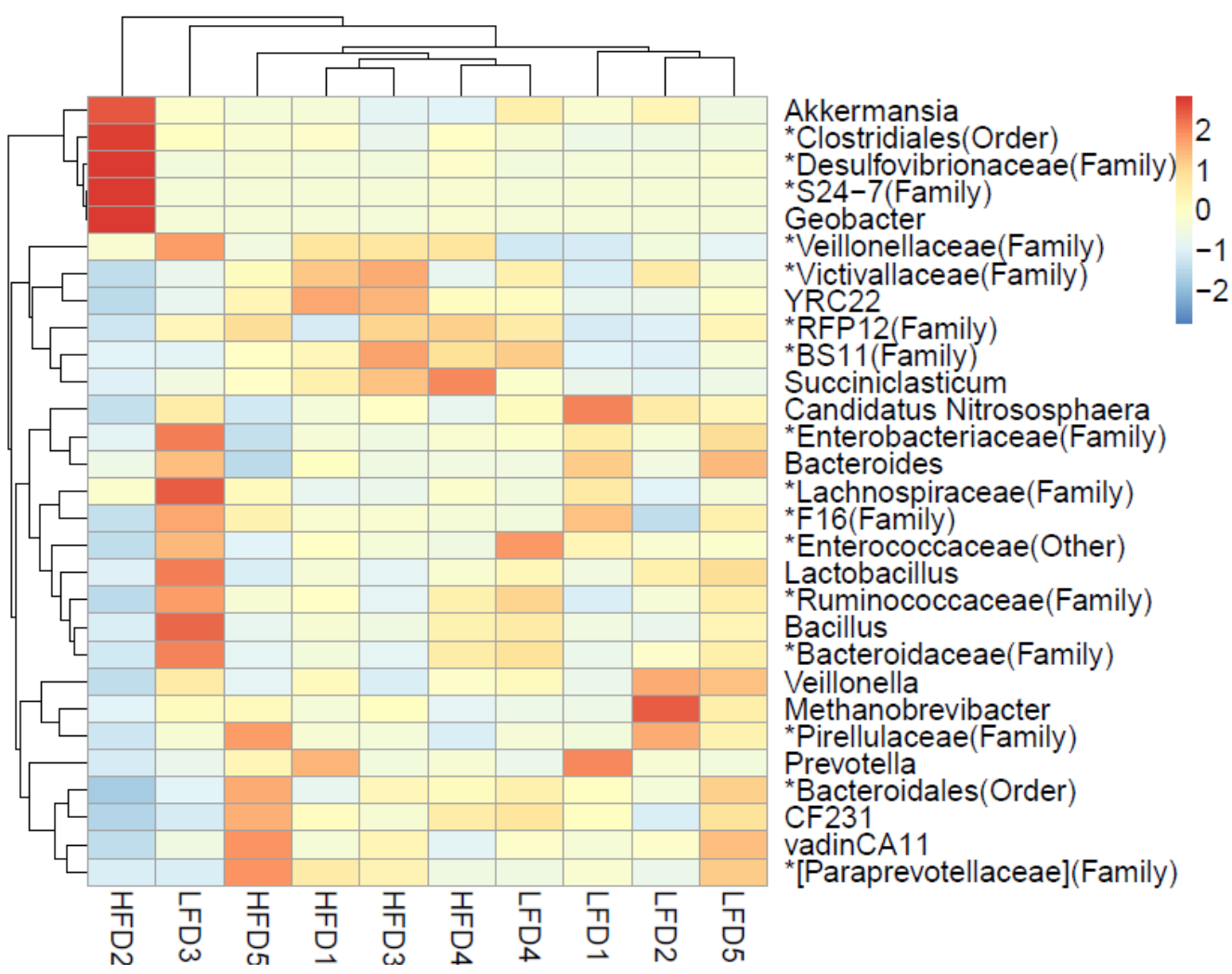

Figure 3. Heatmap of the shared genera between the high neutral detergent fibre digestibility group and low neutral detergent fibre digestibility group.

had focused mainly on bacteria that could secrete cellulolytic enzymes $[2,14]$. However, in this study, the bacteria that had a negative relationship with NDF digestibility was flourished in the rumen. The degree of NDF digestion was likely determined by these "negative" microorganisms rather than cellulolytic bacteria because many of these "negative" microorganisms, such as Enterobacteriaceae, Lactobacillus, and Enterococcus had a much higher relative abundance than the cellulolytic bacteria mentioned above, and the differences of their relative abundance between groups were significant.

In the present study, Enterobacteriaceae was found to be the predominant family both in the two groups (Table 4), and its relative abundance in LFD (5.71\%) was significantly higher than that in HFD (3.52\%). The correlation between Entero-

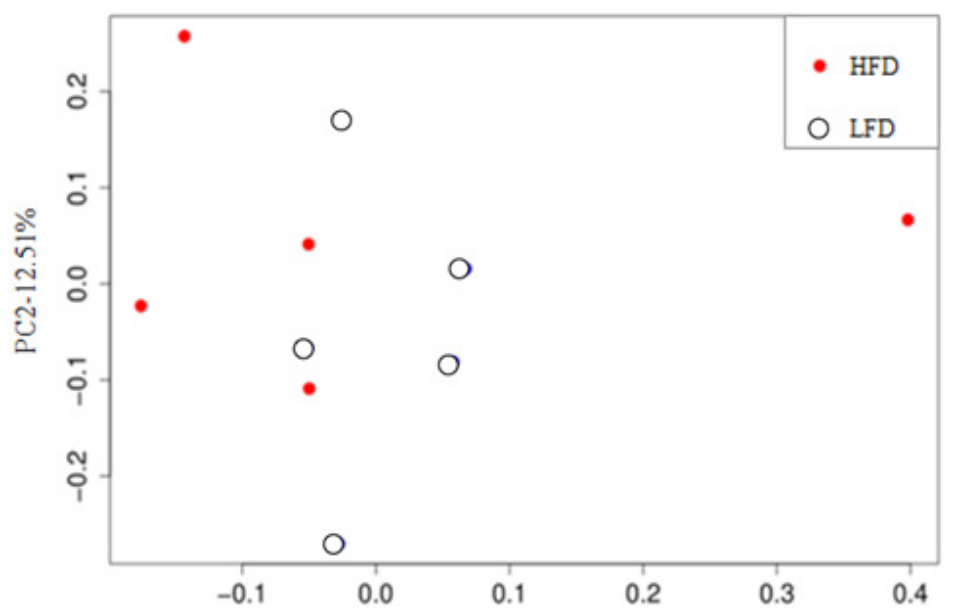

PC1- $14.39 \%$

Figure 4. Principal coordinate analysis (PCOA) plots based on operational taxonomic units (OTUs, grouped at a 97\% sequence similarity threshold) between high neutral detergent fibre digestibility group and low neutral detergent fibre digestibility group. 
Table 5. The correlation between the relative abundance of microorganisms and NDF digestibility

\begin{tabular}{|c|c|c|c|}
\hline & Taxa & $\mathbf{R}$ & pvalue \\
\hline \multirow[t]{2}{*}{ Phylum } & Crenarchaeota & -0.891 & 0.001 \\
\hline & Nitrospirae & -0.733 & 0.016 \\
\hline \multirow[t]{5}{*}{ Class } & Bacilli & -0.491 & 0.15 \\
\hline & Thaumarchaeota & -0.891 & 0.001 \\
\hline & Acidobacteria-6 & -0.745 & 0.013 \\
\hline & Nitrospira & -0.733 & 0.016 \\
\hline & PBS-25 & -0.847 & 0.002 \\
\hline \multirow[t]{10}{*}{ Order } & Enterobacteriales & -0.673 & 0.033 \\
\hline & Lactobacillales & -0.685 & 0.029 \\
\hline & Nitrososphaerales & -0.891 & 0.001 \\
\hline & iii1-15 & -0.661 & 0.038 \\
\hline & Thiotrichales & -0.745 & 0.013 \\
\hline & SBR1031 & -0.879 & 0.001 \\
\hline & MND1 & -0.721 & 0.019 \\
\hline & Nitrospirales & -0.721 & 0.016 \\
\hline & [Entotheonellales] & -0.693 & 0.026 \\
\hline & *PBS-25 (Class) & -0.847 & 0.002 \\
\hline \multirow[t]{13}{*}{ Family } & Enterobacteriaceae & -0.673 & 0.033 \\
\hline & Bacteroidaceae & -0.685 & 0.029 \\
\hline & Nitrososphaeraceae & -0.891 & 0.001 \\
\hline & *iii1-15 (Order) & -0.624 & 0.054 \\
\hline & Sphingomonadaceae & -0.806 & 0.005 \\
\hline & Piscirickettsiaceae & -0.745 & 0.013 \\
\hline & *MND1 (Order) & -0.721 & 0.019 \\
\hline & Ellin517 & -0.661 & 0.038 \\
\hline & *Lactobacillales (Other) & -0.855 & 0.002 \\
\hline & Nitrospiraceae & -0.806 & 0.005 \\
\hline & *Lactobacillales (Order) & -0.709 & 0.022 \\
\hline & SJA-101 & -0.818 & 0.004 \\
\hline & Hyphomonadaceae & -0.685 & 0.029 \\
\hline \multirow[t]{15}{*}{ Genus } & *Enterobacteriaceae (Family) & -0.673 & 0.033 \\
\hline & Enterococcaceae (Other) & -0.721 & 0.019 \\
\hline & Candidatus Nitrososphaera & -0.891 & 0.001 \\
\hline & *Piscirickettsiaceae (Family) & -0.733 & 0.016 \\
\hline & Pseudomonas & -0.709 & 0.022 \\
\hline & *Flavobacteriaceae (Family) & -0.782 & 0.008 \\
\hline & *Ellin517 (Family) & -0.661 & 0.038 \\
\hline & SJA-101 (Family) & -0.818 & 0.004 \\
\hline & Pyramidobacter & 0.661 & 0.038 \\
\hline & *Rhizobiaceae (Family) & -0.818 & 0.004 \\
\hline & *Hyphomonadaceae (Family) & -0.806 & 0.005 \\
\hline & Nitrosopumilus & -0.804 & 0.005 \\
\hline & Paraprevotella & -0.657 & 0.039 \\
\hline & *Cenarchaeaceae (Family) & -0.632 & 0.05 \\
\hline & Dyadobacter & -0.649 & 0.042 \\
\hline
\end{tabular}

NDF, neutral detergent fibre.

bacteriaceae and NDF digestibility had not been reported. Some previous experiments showed that Enterobacteriaceae was a Gram-negative bacteria and contained opportunistic pathogens, such as Escherichia coli and Salmonella, which impaired gut health [25]. Additionally, the research from Sevcik showed that three species of Enterobacteriaceae, including Enterobacter cloacae, E. asburiae, and Klebsiela pneumonia were identified as the source of bovine saxitoxin, which is a paralytic toxin that can block sodium channels in nerves [26]. Up to now, most of studies on Enterobacteriaceae were pathological and pharmacological study and many bacteria in Enterobacteriaceae were considered unprofitable for the host. Accordingly, we inferred that Enterobacteriaceae would be disadvantage for NDF digestibility in ruminants. Nevertheless, this view had not been reported, and further study is needed to confirm it.

The previous study showed that Lactobacillus had a substantial role in lactate metabolism in rumen and there was a positive correlation between its relative abundance and the concentration of lactic acid [27]. In the current study, the relative abundance of Lactobacillus was higher in LFD than that in HFD, and the relative abundance of Lactobacillales increased with the decrease of NDF digestibility through the analysis of correlation. In the rumen, high concentrations of lactic acid led to an imbalance in rumen microorganism flora, and cellulolytic bacteria were more sensitive to the low $\mathrm{pH}$ conditions caused by lactic acid than other bacteria [28]. Furthermore, a research had indicated that subacute ruminal acidosis reduced the digestibility of NDF [29]. Therefore, it was probably due to a high concentration of lactate in the rumen of goats in the LFD group that suppressed the fibrolytic bacteria and further affected the NDF digestibility in this study.

In a previous study, a number of Enterococcus (Enterococcus faecalis, Enterococcus durans, Enterococcus casseliflavus) were identified as cellulase producers in the guts of insects and the colons of humans [30]. This study found the relative abundance of Enterococcaceae and the ${ }^{\star}$ Enterococcaceae (Other) (the bacteria that was not named in Enterococcaceae) in LFD was higher than that in HFD. Enterococcus is one of the mainly genus in Enterococcaceae, and there might some genus in Enterococcaceae have the negative relationship with NDF digestion. Therefore, whether the other bacteria in Enterococcus was related to the degradation of cellulose in the rumen needed further research.

There were also some bacteria whose relative abundance had a significant positive correlation with NDF digestibility, such as Veillonella and Bacteroides (Tables 4, 5). However, the relative abundance of these bacteria in rumen was very low. Therefore, their role in fibre digestion remains unknown and requires further research.

\section{CONCLUSION}

The results obtained in this study indicated that the NDF digestibility was largely different among individual goats and there were significant differences in the structure of rumen microorganisms in goats with different NDF digestibility. 
Additionally, many bacteria had tight correlation with the digestibility of NDF. These results contribute further insights into the rumen bacterial structure under different NDF digestibility conditions and provide evidence for targeted improvement of dietary fibre digestibility in goats

\section{CONFLICT OF INTEREST}

We certify that there is no conflict of interest with any financial organization regarding the material discussed in the manuscript.

\section{ACKNOWLEDGMENTS}

The authors would like to thank the National Key R\&D Program of China (grant number: 2017YFD0502005) and Sichuan Beef Cattle Innovation Team (grant number: 035Z389) for the financial support.

\section{REFERENCES}

1. Hungate RE. Introduction: the ruminant and the rumen. The rumen microbial ecosystem. London, UK: Elsevier Science Publishers, Ltd.; 1988.

2. Russell J, Muck R, Weimer P. Quantitative analysis of cellulose degradation and growth of cellulolytic bacteria in the rumen. FEMS Microbiol Ecol 2009;67:183-97.

3. Stevenson DM, Weimer PJ. Dominance of Prevotella and low abundance of classical ruminal bacterial species in the bovine rumen revealed by relative quantification real-time PCR. Appl Microbiol Biotechnol 2007;75:165-74.

4. Wang Y, Cao P, Lei W, et al. Bacterial community diversity associated with different levels of dietary nutrition in the rumen of sheep. Appl Microbiol Biotechnol 2017;101:3717-28.

5. Niu Q, Li P, Hao S, et al. Dynamic distribution of the gut microbiota and the relationship with apparent crude fiber digestibility and growth stages in pigs. Sci Rep 2015;5:9938.

6. Wang LZ, Qin X, Kong F, et al. Exploring the goat rumen microbiome from seven days to two years. Plos One 2016; 11:e0154354.

7. Xiao DH, Hui YT, Long R, et al. Comparison of methanogen diversity of yak (Bos grunniens) and cattle (Bos taurus) from the Qinghai-Tibetan plateau, China. BMC Microbiol 2012;12: 237.

8. Wang LZ, Zhou ML, Wang JW, et al. The effect of dietary replacement of ordinary rice with red yeast rice on nutrient utilization, enteric methane emission and rumen archaeal diversity in goats. Plos One 2016;11:e0160198.

9. Vigors S, Sweeney T, O'Shea CJ, et al. Pigs that are divergent in feed efficiency, differ in intestinal enzyme and nutrient transporter gene expression, nutrient digestibility and microbial activity. Animal 2016;10:1848-55.
10. Hristov AN, Callaway TR, Lee C, et al. Rumen bacterial, archaeal, and fungal diversity of dairy cows in response to ingestion of lauric or myristic acid. J Anim Sci 2012;90:4449-57.

11. Caporaso JG, Kuczynski J, Stombaugh J, et al. QIIME allows analysis of high-throughput community sequencing data. Nat Methods 2010;7:335-6.

12. Edgar RC. Search and clustering orders of magnitude faster than BLAST. Bioinformatics 2010;26:2460-1.

13. Caporaso JG, Bittinger K, Bushman FD, et al. PyNAST: a flexible tool for aligning sequences to a template alignment. Bioinformatics 2010;26:266-7.

14. Jami E, Israel A, Kotser A, et al. Exploring the bovine rumen bacterial community from birth to adulthood. ISME J 2013;7: 1069-79.

15. Hoover WH. Chemical factors involved in ruminal fiber digestion. J Dairy Sci 1986;69:2755-66.

16. Beauchemin KA, Rode LM, Vjh S. Fibrolytic enzymes increase fiber digestibility and growth rate of steers fed dry forages. Can J Anim Sci 1996;75:641-4.

17. Tan ZL, Lu DX, Hu M, et al. Effect of dietary structural to nonstructural carbohydrate ratio on rumen degradability and digestibility of fiber fractions of wheat straw in sheep. Asian-Australas J Anim Sci 2002;15:1591-8.

18. Nkrumah JD, Okine EK, Mathison GW, et al. Relationships of feedlot feed efficiency, performance, and feeding behavior with metabolic rate, methane production, and energy partitioning in beef cattle. J Anim Sci 2006;84:145-53.

19. Herd RM, Arthur PF. Physiological basis for residual feed intake. J Anim Sci 2009;87:E64-71.

20. Ramos MH, Kerley MS. Mitochondrial complex I protein differs among residual feed intake phenotype in beef cattle. J Anim Sci 2013;91:3299-304.

21. Koch RM, Swiger LA, Chambers D, et al. Efficiency of feed use in beef cattle. J Anim Sci 1963;22:486-94.

22. Kelly AK, Mcgee M, Crews DH Jr, et al. Effect of divergence in residual feed intake on feeding behavior, blood metabolic variables, and body composition traits in growing beef heifers. J Anim Sci 2010;88:109-23.

23. Flint HJ, Bayer EA, Rincon MT, et al. Polysaccharide utilization by gut bacteria: potential for new insights from genomic analysis. Nat Rev Microbiol 2008;6:121-31.

24. Mohammadzadeh H, Yáñez-Ruiz DR, Martínez-Fernandez $\mathrm{G}$, et al. Molecular comparative assessment of the microbial ecosystem in rumen and faeces of goats fed alfalfa hay alone or combined with oats. Anaerobe 2014;29:52-8.

25. Carvalho BF, Aacute Vila CLS, Bernardes TF, et al. Fermentation profile and identification of lactic acid bacteria and yeasts of rehydrated corn kernel silage. J Appl Microbiol 2017;122: 589-600.

26. Sevcik C, Noriega J, D'Suze G. Identification of Enterobacter bacteria as saxitoxin producers in cattle's rumen and surface water from Venezuelan Savannahs. Toxicon 2003;42:359-66. 
27. Coe ML, Nagaraja TG, Sun YD, et al. Effect of virginiamycin on ruminal fermentation in cattle during adaptation to a high concentrate diet and during an induced acidosis. J Anim Sci 1999;77:2259-68.

28. Zhang L, Chung J, Jiang Q, et al. Characteristics of rumen microorganisms involved in anaerobic degradation of cellulose at various $\mathrm{pH}$ values. Rsc Adv 2017;7:40303-10.
29. Huo W, Zhu W, Mao S. Impact of subacute ruminal acidosis on the diversity of liquid and solid-associated bacteria in the rumen of goats. World J Microbiol Biotechnol 2014;30:669-80.

30. Robert C, Bernalier-Donadille A. The cellulolytic microflora of the human colon: evidence of microcrystalline cellulosedegrading bacteria in methane-excreting subjects. FEMS Microbiol Ecol 2003;46:81-9. 